\title{
Presidential Address 2011
}

\section{Challenges in implementing HIV prevention interventions among high risk groups in Sri Lanka}

\section{Chandrika Wickramasuriya ${ }^{1}$}

Our chief Dr. Ranjababu Kulasegaram.

Founder President Dr. Lucian jayasuriya

Members of the council Fellows of the college,

Members of the college

Family members of Late Dr. G.N. Jayakuru

Distinguish invitees.

In my address today at the inauguration of the $16^{\text {th }}$ annual academic sessions of the Sri Lanka College of Venereologists. I decided to focus my brief address on challenges in implementing 'HIV the prevention interventions among high risk groups in Sri Lanka'

Three decades to the HIV epidemic where advancement of anti retroviral therapy has made this once deadly disease in to another chronic illness, where people in the developed world are now talking about treatment as prevention which is very costly (secondary prevention), we in the developing world continue to emphasise on primary prevention, in responding to HIV which is cost effective.

Over the past 25 years Sri Lanka has continued to remain one of the few countries in the region with a low level HIV epidemic. HIV prevalence in the general population continued to be less than $1 \%$ and among the high risk populations less than 5\%. By end of June 2011 of the estimated 3000 cases, only 1388 infected persons have been detected. Of that $60 \%$ were men and $40 \%$ were women. Fifty children were also detected and 14 are on treatment.

How did these people get infected?

$83 \%$ were due to heterosexual, $11 \%$ due homosexual and $4.4 \%$ were due mother to child transmission. Therefore, going by the current prevalence can we be complacent? Will this low HIV prevalence remain low? Let us see the trend of case detection over the last 25 years. It shows a steadily rising trend and it is noteworthy that during last five years number of infections detected in 20$24 \mathrm{yr}$ age group is gradually increasing.

Is there any warning system that gives directions towards a possible epidemic in low prevalence countries? The answer is YES

It has shown that the Behavior is the primary early warning system in low prevalence countries:

Behavioral information can indicate the populations are at risk locally, and can suggest pathways the infection might follow if nothing is done.

\section{What are these behaviours that could influence the growth of an epidemic?}

They are:

- having multiple partners

- the presence of overlapping or concurrent sexual partnerships

- $\quad$ size of populations with multiple partners

- sexual mixing patterns of these high risk populations with the general population

- coverage of prevention interventions among high risk populations

- $\quad$ and the extent of protective behaviours (use of condoms) among them

Who are the population with such behaviors?

They are

- Female Sex Workers and their clients

- Males with same sex partners (MSM) 
- Injecting drug users who share contaminated needles and syringes

\section{What is the situation in Sri Lanka?}

The two External Reviews carried out in 2006 and 2011 on the National Response to HIV during last 10 years showed that there is considerable evidence that Sri Lanka is vulnerable to the development of a concentrated HIV epidemic due to the presence of behavioural factors which have shown to lead the epidemic in other countries in the Asian region.

Up to what level do these behavioural factors exist in Sri Lanka?

I am going to focus only among female sex workers and MSM since injecting drug use currently seems to be low in Sri Lanka .

\section{Let us examine the size of the female sex worker population and their behaviour.}

The national estimate in 2010 gave the figure of 35,000 - 47,000 female sex workers in Sri Lanka.

They operate on average 3-5 days a week. Mean number of clients on last working day was 3-2 per day. Their consistent condom use with paying clients were $>60 \%$ in all categories except casino based sex workers.

If we take the minimum number of partners as 2 on average, the estimated 35000 to 47,000 , sex workers would have sexual relations with 70,000 to 94,000 male clients per day.

Therefore if the extent of condom use does not reach high levels and is maintained at high levels in all categories of sex workers there is a potential for the epidemic to spread among the clients of sex workers, who in turn will spread the infection to their wives and through them to their children

Now I would like to discuss same sex behavior among men

Men who have sex with men has become a significant group in driving the HIV epidemic in Asia. Currently they represent a major source of new infections in many Asian countries. In some countries in Asia HIV infection has spread among MSM rapidly. Within a period of two years in Bangkok, Thailand, the prevalence has increased from $17.3 \%$ to $28 \% \%$, in Beijing, China a six fold increase is seen and in Karachchi, Pakistan it has doubled.

It is reported that in Asia, MSM are disproportionately affected by the HIV epidemic, with the odds of MSM having HIV infection being 18.7 times higher than that in the general population. The Asian Epidemic Model projects that unless effective prevention measures are intensified, by 2020 , around $46 \%$ of new infections in Asia will be among MSM.

\section{What is the situation of MSM population in Sri Lanka and their behavior?}

The National estimate for MSM in 2010 was 24,000 to 37,000 . Their rate of partner change during last 12 months was reported to be 09 on average. Among MSM, beach Boys (BB) forms a noteworthy sub group.

Beach boys refers to young men who work on and around the beaches and who offer transactional sexual services to men and women. The available estimate for beach boys is 30,000 (UNDP 2007) and $46 \%$ of $\mathrm{BB}$ are reported to have sex with male partners and 14,000 BB are identified as MSM.

When we studied the sexual behavior of MSM, $83 \%$ had non-regular male partners, while $68 \%$ also had regular partners. They also had regular and nonregular female partners.

It is reported that same sex monogamy is not common in Asia due to social taboos and discrimination, and therefore a fair number have sex with women and some of them are married.

\section{Beach Boys}

Beach boys are mainly heterosexuals. $71 \%$ had a regular female partner, 53\% had non regular female partners but a significant number of them have male 
partners too. There is over lapping sexual partners among MSMs and beach boys in Sri Lanka. They have regular and non-regular male partners as well as female partners.

And beach boys have both local and foreign partners.

Condom use is the most effective method of protection against HIV in these groups with multiple partners. Condom use with regular partners has been noted to be very low in both groups - MSM -32\% and beach boys- $31 \%$. Condom use with non regular partners is also not satisfactory in both groups. MSM $-51 \%$ and beach boys- $64 \%$. Condom use with female partners was very unsatisfactory among both groups. Hence, there is a significant population of MSM with high risk behavior in Sri Lanka. Their level of condom use is very low. Therefore there is a potential for the HIV epidemic to grow among them.

Despite this evidence, open discussion on MSM is restricted due to socio-cultural reasons, preventing introduction of effective prevention interventions.

When we analysed how the last 100 HIV cases reported to the National STD/ AIDS Control Programme contracted the infection during the past three years, it was revealed more MSM than female sex workers were within these 100 cases in each year.

You might wonder why I am not concerned about the $43 \%$ of transmission due to casual sex.

Existing evidence shows that in Asia because relatively few women have more than one partner and casual sex is unlikely to drive the epidemic.

Further analysis showed that in 2009, 62 out of 100 were males, of these, 21 were MSM.

MSM accounted for $33 \%$ of new infections among males in that year. In 2010, of the last 100 cases, 68 were males, and of these 18 were MSM. MSM accounted for $26 \%$ of new infections among males in 2010. In 2011, 58 out of 100 were males, of these, 22 were MSM. MSM accounted for $38 \%$ of new infections among males.
As you can see MSM accounted for more than 25\% of the reported HIV infections during last three years.

Ladies and gentlemen, so far I have attempted to present some behavioural risk factors in the high risk population groups who have the potential to drive the HIV epidemic in Sri Lanka.

We know behavior is the early warning sign.

\section{What are the effective prevention interventions for these groups who are driving the epidemic?}

Based on an analysis of existing best practices in the region, minimum elements of a standard preventive package for Female Sex Workers, MSM and IDUs have been identified. This package includes:

- Outreach through peers providing education on Sexually transmitted infections/HIV and development of skills to change behavior to reduce sexual risk,

- promote and provide condoms,

- link high risk groups with STI care

- creating an enabling environment to carry out these interventions successfully and strengthening the capacity of civil society organizations working for them.

\section{Effective prevention takes time.}

It has shown that effective prevention through behavior change does not happen overnight. It takes time. The intervention that has shown to give results during a short period is condom promotion. If $60 \%$ of theses high risk populations practice $100 \%$ condom use, the growth of an epidemic can be halted. To achieve this level of condom use the coverage among high risk groups has to be more than $80 \%$.

But implementation of such prevention interventions to these groups because have become a challenge in low prevalence countries due to several reasons. 


\section{Low prevalence is seen as a low priority}

Low HIV prevalence is used to support the mistaken belief that the behaviors that promote HIV transmission do not exist in the country and used as a justification for non-response. Sometime low prevalence leads to a rather complacent view that social norms and mores would hold HIV in check in those countries. In most countries these behaviors are socially not accepted and are illegal. Therefore they live as a hidden populations making access difficult.

In Low HIV prevalence countries where unfavorable societal attitudes prevail toward these populations, the tendency is to produce a default prevention strategy targeting the masses that almost totally bypasses these sub-populations with greater risk behavior. This is because such responses are less threatening and more publicly rewarding even though they are less effective at preventing epidemic growth.

Yet prevention efforts to high risk groups must begin before HIV prevalence grows to measurable levels if countries hope to prevent an epidemic in low prevalence state.

What is the strategy for reaching these populations?

- Peers led approach is considered as the most practical and cost effective strategy which has shown successful results in controlling the epidemics in the region.

Carrying out such interventions to these groups is a real challenge in Sri Lanka.

- Sex work and MSM behaviour are illegal and not accepted due to socio cultural reasons.

- These Sub populations live underground making access for preventive interventions difficult and only a small proportion of them access services.
- Health care providers cannot reach these hidden populations in the community to increase coverage of preventive interventions.

- The practical way to access these hidden population in the community is through their peers who are working with them.

- Therefore, it is necessary that all relevant stakeholders have a better understanding about the value of early action and

- Effectively support these preventive activities through their active participation

Ladies and gentlemen I like to end by reiterating that

'Current HIV prevalence is not predictive of the future of the epidemic. It is the current behavior patterns that predict the future of the epidemic' 